\title{
AKTIVIERENDE METHODIK IM FREMDSPRACHENUNTERRICHT - Nutzen, Wirkung, praktische Beispiele
}

\author{
Daniela Schultz, Martin Bradbeer, Virginie Bimont, Greg Bond, Adrian Stiglbauer
}

\section{Zusammenfassung}

„Spielen im Unterricht macht Spaß, aber dafür fehlt uns die Zeit, wir haben in einem Semester so viel Stoff zu bewältigen, dass wir kaum Zeit für Spiele haben, außerdem finden die Studierenden das eher kindisch. "Im Folgenden zeigen wir anhand theoretischer Überlegungen und praktischer Beispiele, dass man gerade bei spielerischen, aktivierenden Methoden lernt. Im ersten Teil dieses Beitrags werden theoretische Grundlagen für das aktivierende, spielerische Lernen vorgestellt. Im zweiten Teil werden methodische Grundsätze präsentiert, und im dritten und letzten Teil berichten wir von praktischen Beispielen aus unserem Unterricht.

Die Tatsache, dass die Beispiele dem Fremdsprachenunterricht entnommen sind, bedeutet jedoch nicht, dass aktivierende Methodik nur dort sinnvoll eingesetzt werden kann. Vielmehr hat sie einen allgemeinen Nutzen für die Hochschuldidaktik in allen Fachgebieten. Gerade heute, wo Lernziele an Hochschulen zunehmend nicht nur über den "Stoff“ definiert werden, sondern kompetenzorientiert sein und die Autonomie der Lernenden fördern sollen, können aktivierende Methoden in jedem Fachgebiet und in jeder Art von Lehrveranstaltung bedeutsam sein.

\section{Abstract}

This article looks at contemporary methods for language teaching at university level, and for all languages at all levels from beginners to advanced. The article aims to show that activating, playful methods with an emotional and practical point of reference are most effective in today's language teaching. Moreover, we claim that problem-solving and participative methods are not only relevant for language teaching, but for all teaching, and especially at university level, where the relevance of materials and methods for students must be highly evident, and students need to discover effective paths and ways through a large amount of material.

The first part looks at the theoretical background to participatory teaching and learning, considering learning styles and offering explanations as to what is meant by playful teaching methods and why they are effective. Memorability, sensuality and experiential learning are key ideas here. The second part of this essay looks at some of the methods in teaching that are appropriate to experiential learning. In the final part of this article we present concrete examples of playful and participatory learning from our own teaching experience.

\section{»I. EINLEITUNG:}

\section{THEORETISCHE ÜBERLEGUNGEN}

1. Voraussetzungen für gelingendes Lernen

Was bedeutet es, etwas zu lernen? Lernen bedeutet sicher nicht, einen Vortrag anzuhören, Wissen zu rezipieren. Lernen bedeutet auch nicht, etwas zu verstehen. Lernen bedeutet, Wissen aufzunehmen und aktiv zu verarbeiten, so dass man langfristig mit dem Erlernten umgehen kann. Lernen bedeutet somit eine Veränderung des Erlebens und Verhaltens aufgrund von individuellen Erfahrungen mit und in der Umwelt.

"Lernen", sagte der Hirnforscher Gerald Hüther im Februar 2012 auf der weltweit größten Bildungsmesse „Didacta“ in Hannover, können die Lehrenden „nicht machen, nicht erzwingen, sie können nur den Rahmen dafür schaffen, damit Lernen gelingt - einen Raum gestalten, in dem sich das Gewünschte ereignet" (Tagesspiegel
16.2.2012, 29). Wenn wir wollen, dass etwas gelernt wird, müssen wir den Lernenden die Möglichkeit geben, das neue Wissen in das vorhandene Weltbild zu integrieren bzw. es neu zu konstruieren und das eigene Erleben und Verhalten darauf neu auszurichten.

Daraus ergeben sich einige Schwierigkeiten für Lehrende: Erstens ist jeder Mensch anders, „keiner ist wie der andere“ (Asendorpf 1988: 203). So banal diese Einsicht ist, so bedeutsam ist sie für den Unterricht. Es gibt nicht nur verschiedene Lerntypen (vereinfachend werden oft visuelle, verbalabstrakte, auditiv-kommunikative und haptische Lerntypen unterschieden), sondern Lernende sind alle Individuen mit individuellen Denkstrukturen und unterschiedlicher Herkunft, die für unterschiedliche Inhalte unterschiedliche Verarbeitungsmöglichkeiten bevorzugen. Wird der zu lernende Stoff mit den bereits vorhandenen Erfahrungen verknüpft und ist dort langfristig verfügbar, können wir behaupten, dass er gelernt wurde.

2. Die Speicherung des Erlernten Es gibt verschiedene Erklärungsansätze in der Gedächtnisforschung; die meisten Theorien gehen von einem Modell mit drei unterscheidbaren Stadien im menschlichen Gedächtnis aus (Roche 2008: 18). Demnach verfügen wir über einen sensorischen Speicher (das Ultrakurzzeit-Gedächtnis), ein Kurzzeit-Gedächtnis und ein LangzeitGedächtnis.

Der sensorische Speicher registriert schnelle Reize, die Dauer der Speicherung beträgt Zehntelsekunden bis einige Sekunden. Das Kurzzeit-Gedächtnis funktioniert wie eine Art Arbeitsspeicher, hier wird "geistige Arbeit" verrichtet und hier werden Informationen nach dem Grad ihrer Bedeutsamkeit selektiert (Lehner und Ziep 1997: 127133).

Die Kunst des Lehrenden besteht laut Hüther darin, etwas für Lernende „bedeutsam zu machen, was ihnen 
auf Deutsch gesagt, am Arsch vorbei geht" (Tagesspiegel 16.2.2012, 29). Ist die Information dann so bedeutsam geworden, dass sie langfristig abrufbar sein soll, so befindet sie sich in der „Wiederholungsschleife" und wenn sie häufig genug wiederholt wurde, gelangt die Information in das Langzeit-Gedächtnis. Dort ist sie dann dauerhaft verfügbar. Vergessen bedeutet für das Langzeit-Gedächtnis nur, dass die Information nicht auffindbar ist. Das Schwierigste beim Erinnern ist also der Zugriff, der Zugang zur gesuchten Information.

Was bedeutet das nun für die Lehre?

1. Damit der Lernstoff den "Filter“, das sensorische oder UltrakurzzeitGedächtnis passiert, muss er für die Lernenden ein hohes Maß an Bedeutsamkeit aufweisen.

2. Dafür, dass die Informationen, die ins Kurzzeit-Gedächtnis gelangt sind, nicht wieder verloren gehen, ist es wichtig, den Stoff zu wiederholen. Dies sollte auf möglichst vielfältige Art geschehen, so dass möglichst viele verschiedene Eingangskanäle aktiviert werden.

3. Um die Informationen aus dem Langzeit-Gedächtnis problemlos abrufen zu können, ist es notwendig, dass sie bereits bei der Informationsaufnahme in organisierten, geordneten Strukturen vorliegen und in bereits vorhandenen Kontexten aufgenommen werden (Lehner und Ziep 1997: 132).

\section{Emotionen und Lernerfolg}

Der enge Zusammenhang von Lernvorgängen und Emotionen wird besonders deutlich, wenn wir uns selbst an Dinge erinnern, die von hohem persönlichem Wert sind. An Sachverhalte, die nicht nur auf kognitiver, sondern auch auf emotionaler Ebene verarbeitet werden, können wir uns besonders gut erinnern.

Für ein vollständiges Spracherwerbsangebot ist daher zu berücksichtigen, dass bei den folgenden Aspekten eine emotional positive Haltung zum Unterrichtsgeschehen von herausragender Relevanz für den nachhaltigen Lernerfolg ist.
Vielfalt des Lernangebots: inhaltsorientierte und sprachformorientierte Verarbeitung, Sprachproduktion und Angebot von Kompensationsstrategien, das sind Strategien, die den Studierenden helfen, sprachliche Defizite auszugleichen

Multisensorische Informationsdarbietung (verschiedene Lernkanäle)

Verbindung von logisch-analytischen und intuitiv-kreativen Prozessen

Schaffung eines positiven Lernklimas (siehe auch Lehner und Ziep 1997: 143), was nur erreicht werden kann, wenn wir die Lernenden als Partner respektieren und ernst nehmen, ihre Fragen, Bedürfnisse und Interessen berücksichtigen und so einen respektvollen Umgang miteinander etablieren

\section{» II. METHODISCHE GRUNDSÄTZE DES AKTIVIERENDEN LERNENS}

\section{Das Lernsandwich}

Lehrende sollten mit den Lernenden zusammen einen Raum schaffen, in dem die Lernenden ihr neu erworbenes Wissen in individuell verändertes Erleben und Verhalten umsetzen können. Um den Studierenden dazu Gelegenheit zu geben, bedeutet das konkret, dass wir präsentierende Unterrichtsphasen stets in aktive Phasen einbetten müssen.

Schon der Begriff "Lernsandwich" macht deutlich, dass gute Lehrende den Studierenden appetitlich zubereitete Häppchen servieren sollten und zwar aus den Zutaten, die von oder in Abstimmung mit ihnen ausgewählt wurden. Außerdem bedeutet Sandwich, dass leckere Zutaten in einer bestimmten Reihenfolge angeordnet werden sollten:

\section{Einstieg/Einführung}

Informationsaufnahme

- rezeptives Lernen

Informationsverarbeitung - aktives Lernen
Informationsaufnahme

- rezeptives Lernen

\section{Zusammenfassung/Ausstieg}

(nach Lehner und Ziep 1997: 18)

Diethelm Wahl konstruiert eine etwas komplexere Variante eines Lernsandwichs, bei dem auch die Schnittstellen oder Übergangsstellen zwischen rezeptivem Lernen, das er die „Phase der kollektiven Vermittlung" nennt, und aktivem Lernen, das bei ihm „Phase der subjektiven Auseinandersetzung“ heißt, betrachtet werden. Diese Stellen, er nennt sie "Gelenkstellen“, dienen der Transparenz und appellieren an die Autonomiefähigkeit der Lernenden. Besonders an der ersten Gelenkstelle, die oben als Einstieg/Einführung bezeichnet wurde, aber auch an den anderen Gelenkstellen, soll ein Austausch über den Ablauf der Lehrveranstaltung stattfinden, Redeschwellen sollen so überwunden werden, Interessen und Vorkenntnisse sollen erfasst werden. So kann das Lernen an den Voraussetzungen der Lernenden ausgerichtet und zu einem aktiven, selbst gesteuerten Prozess werden (Wahl 2006: 96-104; siehe auch die hierzu gut passende Methode des „taskbased language teaching" (vorgestellt von O'Donoghue 2012).

\section{Stoffreduktion}

Nur wenn alle Inhalte vollständig präsentiert werden, z. B. möglichst viele Folien gezeigt, oder jede Lehrbuchseite bearbeitet wird, können sich Studierende das notwendige Wissen aneignen - so eine verbreitete Haltung in der Hochschule. Der Stoff muss geschafft werden. In Anbetracht der knappen Ressource Zeit und einer großen Lehrstofffülle sollten die Inhalte eher so reduziert werden, dass sie exemplarischen und prototypischen Charakter haben, so dass die Studierenden auch mithilfe erlernter Kompensationsstrategien das Erlernte rekonstruieren, wiedergeben, anwenden und umsetzen können. Gute Lehrende sind „gute Fachmänner" auf ihren Gebieten, nicht nur weil sie über umfangreiches Wissen verfügen, sondern vor allem, weil sie wissen, was wirklich wichtig und wesentlich ist und in eine assoziative Struktur eingefügt werden kann, d. h. an schon Bekanntes angeknüpft 
werden kann (Lehner und Ziep 1997: 28-31).

\section{Spiele als Simulationen authentischer Kommunikations- situationen}

Dass sich für das im Lernsandwich aufgeführte Lernen aktivierende und spielerische Methoden besonders gut eignen, möchte ich hier zeigen.

Die Begriffe "aktivierende Methodik“, "spielerische Methoden“ und „Spiele“ werden häufig nebeneinander verwendet, weil die Grenzen fließend sind. Es geht um Methoden, die Lernende dazu zu bewegen, aktiv zu handeln und zu kommunizieren. Die kognitionspsychologische Forschung stellt fest, dass wir den Lernstoff desto länger behalten, je lebendiger er gestaltet wird (Lehner und Ziep 1997: 15-19). Aktivierende, spielerische, lebendige Methoden dienen nicht nur der Auflockerung der Unterrichtsatmosphäre, sondern sie sind authentische Kommunikation, weil Spiele immer ein Ziel haben, das nicht die Sprache oder der zu lernende "Stoff“ selbst ist. Die Studierenden beteiligen sich nach vorgegebenen Regeln am Spiel- bzw. Unterrichtsgeschehen und setzen dafür die erlernte Sprache oder den erlernten Stoff ein. Damit ist eine reale Situation gegeben, in der die Studierenden keine Übung absolvieren, sondern die zu lernenden Mittel einsetzen, um etwas zu erreichen.

Im besten Fall wird dabei gelernt, ohne dass die Teilnehmenden es merken. Auch die bei dieser Methode häufig zu findende hohe Emotionalität und an persönliche Erlebnisse geknüpfte Lernerfahrung sind gute Voraussetzungen für den Lernerfolg. Vorteilhaft ist auch die hohe Partizipation, die ganze Gruppe muss aktiv sein: Nach einer bestimmten Reihenfolge kommen alle, auch eher zurückhaltende Studierende, an die Reihe und haben die Möglichkeit, sich zu beteiligen.

\section{Partizipation der Lernenden fordern}

Es ist keine neue Erkenntnis, dass Frontalunterricht, bei dem die Lehrenden dozieren und die Studierenden zuhören und sich evtl. Notizen machen, häufig nicht den gewünschten Lernerfolg erzielt. Heutzutage weiß man oft nicht, was die Lernenden während der
Vorlesung mit ihren aufgeklappten Notebooks veranstalten - aber man kann sicher sein, dass sie nicht mit voller Aufmerksamkeit beim Thema der Vorlesung sind.

Bei der aktivierenden Methodik kann auf diese Situation weitestgehend verzichtet werden. Die Studierenden werden von Anfang an eingeladen, den Lernprozess selbst aktiv zu gestalten. Über die aktive Mitarbeit hinaus dürfen die Studierenden in den Unterrichtsablauf eingreifen und ihre Vorschläge zum Verfahren einbringen. Phasen der kollektiven Vermittlung können von Studierenden selbst gestaltet werden, in Form einer Präsentation, einer Demonstration oder einer von Studierenden eingebrachten spielerischen Übung. Von kollektiver Vermittlung wird hier gesprochen, weil im Gegensatz zur Phase der aktiven, subjektiven Auseinandersetzung hier alle Lernenden hinsichtlich Form und zeitlichem Verlauf das Gleiche tun (Wahl 2006: 105). Die Lernenden verfallen nicht in eine Konsumhaltung, in der sie meinen, „nur“ zum Unterricht kommen zu müssen, während der Lehrende oder das Lehrwerk das Lernen für sie „übernehmen".

In regelmäßigen Abständen wird reflektiert, welche Unterrichtsaktivitäten, Übungen, Spiele, Themen usw. erfolgreich waren und welche wiederholt werden können. Das heißt, dass die Studierenden immer wieder selbst ihre Lernziele formulieren können und auch den Weg dahin besprechen sollten.

\section{Sinnlichkeit und Bewegung}

Langes Sitzen am Tisch und Zuhören ist schnell ermüdend. Durch den Einsatz von spielerischen Methoden und die Berücksichtigung aller Sinne beim Lernen entsteht Bewegung im Seminarraum, es wird etwas gemacht. Schon kleine Bewegungen, beispielsweise das kurze Aufstehen und Wechseln des Platzes für eine Partner- oder Gruppenübung, fördern die Konzentration und aktivieren neue Energien. Vom Lehrenden erfordert manch aktivierende Methodik zunächst Mut, neue Lehrsituationen zu initiieren, denn wenn man vom herkömmlichen, bekannten Weg abweicht, stößt man mitunter auf Widerstände der Studierenden. Allerdings handelt es sich hier um Unsicherheit, die sich schnell legt, wenn die Studierenden erkennen, dass sie nun aktiv, praxisorientiert und vor allem mit hoher Relevanz arbeiten.

Der hier postulierte positive Effekt aktivierender Methodik auf den Lernerfolg soll im folgenden praktischen Teil exemplarisch mittels Beispielen illustriert werden. Aktivierende Methoden, Spiele etc. wurden hierbei hinsichtlich verschiedener Zielgruppen und unterschiedlicher Lernziele eingesetzt.

\section{» III. PRAKTISCHE BEISPIELE AUS DEM FREMDSPRACHENUNTERRICHT}

Die Schaffung einer effektiven und aktivierenden Lernatmosphäre im Unterricht

\section{Zielgruppe:}

alle Studierenden, die an einem Sprachkurs teilnehmen

\section{Lernziel:}

Jeder hat seine eigene Art des Lernens, und es ist sehr wichtig für Studierende, gleich zu Beginn eines Kurses zu diskutieren, zu hinterfragen und herauszufinden, welcher Lernertyp sie sind und was sie individuell im Unterricht tun können, um ihre Lernerfahrungen zu verbessern.

\section{Methode:}

Normalerweise werden sich die Studierenden und die Lehrenden in der ersten Unterrichtsstunde vorstellen und über den Kurs sprechen. Die erste Unterrichtsstunde sollte auch dazu dienen, Methoden vorzustellen, wie man am besten während des Unterrichts lernt. Die Methode, um über die Vielfalt der Wege, wie Menschen lernen, zu reflektieren, ist eine Mischung aus Selbstreflexion, Partnerarbeit, Gruppenarbeit, Brainstorming und Diskussion.

Um die Studierenden zu aktivieren, können die Lehrenden zuerst die Lernenden in Paaren oder kleinen Gruppen fragen, wie sie lernen möchten, ob sie sich Notizen machen, ob sie den Unterricht z. B. mit ihren Handys aufzeichnen, wie sie sich für eine Prüfung vorbereiten, was sie gerne in einem Sprachkurs tun. Sie sollten auch darüber diskutieren, was sie nicht mögen! Die Lehrenden sollten dann Beispiele herausgreifen und an die Tafel (Karten und eine Pinnwand können auch verwendet werden) die folgenden 
drei Überschriften schreiben: visuell, auditiv und taktil. Die Studierenden sollen dann entscheiden, welche Strategie/Übung/Tätigkeit/Spiele welchem Lernstil entsprechen. Diese Aktivität wird nicht nur die Studierenden motivieren zu überlegen, welcher Lerntyp sie sind, sondern auch die Diskussion über Lernstrategien fördern. Sobald dies geschehen ist, sollten die Lehrenden die Studierenden fragen, was sie individuell im Klassenzimmer tun können, um ihre Lernerfahrung zu optimieren. Im Folgenden werden einige nützliche aktivierende Strategien aufgeführt, die in der ersten Unterrichtsstunde festgelegt und eingesetzt werden können, um die Sprachkenntnis zu verbessern:

1. Viele Studierende sind besorgt, dass sie die Fehler von ihren Kommilitoninnen und Kommilitonen übernehmen. Aber auch das Gegenteil ist der Fall: Studierende können eine Menge von den anderen Studierenden lernen: Jede Studentin und jeder Student wird in das Klassenzimmer mit einer ganz eigenen Kenntnis der Sprache kommen. Die Lernenden sollten ermutigt werden, zu hinterfragen, was ihre Mitstudierenden sagen und die Lehrenden fragen, ob es richtig ist. Das fördert auch die Diskussion in der Klasse und das Lernen.

2. Die Studierenden sollten Fragen an die anderen Studierenden in ihren Köpfen beantworten. Wenn sie das Gleiche sagen würden und es richtig ist, dann ist alles in Ordnung. Wenn sich ihre Antwort unterscheidet, sollten sie mit den Lehrenden überprüfen, ob ihre Version richtig ist.

3. Wenn die Studierenden neue Worte oder eine neue Phrase lernen, sollten sie diese laut sagen (aber auch nicht zu laut!).

4. Die Studierenden sollten Notizen in der neuen Sprache aufschreiben und Beispielsätze machen, um die Sprache im Kontext zu lernen, sie sollten Definitionen usw. notieren, aber sie sollten versuchen, sie nicht in ihre Muttersprache zu übersetzen.

5. Die Studierenden und Lehrenden sollten sich auf eine bestimmte Art der Korrektur im Klassenzimmer einigen. Weil Lernen ein Prozess ist, sollte der
Umgang mit Fehlern auch ein Prozess sein. Die Studierenden sollten zuerst versuchen, die Fehler selbst zu korrigieren, wenn dies nicht möglich ist, sollten andere Studierende, Peers, versuchen zu korrigieren, die Lehrenden sind die letzte Option.

6. Schließlich sollten die Studierenden aufgefordert werden, sich keine Sorgen über Fehler zu machen. Sie sind in einer Lernatmosphäre, in der sie keine Angst haben müssen, Fehler zu machen! Jeder lernt aus Fehlern und wenn die Studierenden keine Fehler machen, sprechen sie entweder nicht oder die Aufgaben sind viel zu einfach!

\section{Zusammenfassung:}

Bis zum Ende des Unterrichts sollten die Lehrenden und Studierenden diese Lernstrategien und die Wichtigkeit, sie während des Unterrichts zu verwenden, im Detail diskutiert haben. Dieser ganze Prozess ist äußerst nützlich beim Aufbau einer motivierenden und Erfolg versprechende Lernatmosphäre und bei der Schaffung einer positiven Dynamik innerhalb der Klasse. Der Unterricht wird von nun an Spaß machen, aktivierend, effektiv und lernerzentriert sein!

Spielerische und kreative Erlebnisse als aktivierende Methode im Sprachunterricht

\section{Zielgruppe:}

Studierende jeden Alters, Anfänger, die Gruppe umfasst 3 bis 16 Studierende

\section{Lernziele:}

- die Studierenden haben eine positive Erfahrung mit der Fremdsprache

- Entspannung beim Lernen - gute Lernatmosphäre - die Fremdsprache ist das Mittel, um etwas zu erreichen, was nicht nur das Erlernen der Sprache selbst ist -alle fünf Sinne und alle Fähigkeiten werden eingesetzt - die anderen Studierenden kennenzulernen und mit ihnen zu agieren und auf sie zu reagieren - neues Vokabular zu lernen und die Syntax zu ordnen, zu wiederholen und zu lernen

\section{Methode:}

Die Lehrenden präsentieren in der Fremdsprache das Material, mit dem später gespielt wird, sie erklären die Regeln mit vielen Beispielen, sie agieren zuerst selbst gleichzeitig mit Sprache und Bewegung. In einer zweiten Phase werden die Studierenden aktiv, sie werden zu Schauspielern. Die Lehrenden unterstützen die Studierenden: sie loben, helfen, korrigieren und verbinden dabei immer die Sprache mit der Bewegung oder Aktion. In einer dritten und letzten Phase sind die Lehrenden nicht mehr aktiv, sie beobachten die Studierenden, die zusammen kommunizieren.

\section{Sieben Familien: ein Kartenspiel}

Dieses Kartenspiel wird mit "sieben Familien" a sechs Karten gespielt. Die Karten werden zuerst von den Lehrenden und später von den Studierenden hergestellt bzw. mitgebracht. Es wird geschrieben, gezeichnet, geschnitten, gebastelt usw. Das Material und die Aktionen werden in der Fremdsprache benannt, auch wenn die Lexik komplizierter und die Syntax der Anweisungen komplexer wird. Die Karten werden gemischt, ein Stapel steht in der Mitte, und alle Mitspielenden bekommen fünf Karten. Das Ziel: So schnell wie möglich so viele Familien wie möglich zu sammeln. Ablauf: Man muss einen Spielenden nach einer Karte fragen, wenn sie/ er diese Karte hat, bekommt man diese Karte und darf weiterspielen, wenn nicht, nimmt sie/er eine Karte von dem Mittelstapel und die/der nächste ist an der Reihe. Man darf nur nach Familien fragen, von denen man schon mindestens eine Karte hat.

Zum Beispiel: „eine sportliche Familie“ (der Vater spielt Fußball, die Mutter schwimmt...), "hast du den Vater, der Fußball spielt?“ - „sieben Familien“ mit Verben ( 7 verschiedene Verben werden konjugiert, man erhält 6 Karten mit den verschiedenen Personalpronomen und Endungen), „Hast du „wir gehen“?“

Mit diesen Karten können die Studierenden viele verschiedene Themen- und Grammatikbereiche üben, sie sprechen über Themen wie Freizeit, Familie, Länder, Berufe usw. und üben Verbkonjugation, Tempus, Adjektive und Satzbau usw. Sie lernen Fragen zu stellen und zu beantworten und Vokabular zu klassifizieren.

\section{Kritisches Resumée:}

Das Spiel eignet sich bestens, um eine Sprache zu lernen. Es kommt zu emotionaler Stimulierung und zu spannenden Erlebnissen, zu automatischer 
Wiederholung, es werden verschiedene Materialien genutzt, Selbstvertrauen wird entwickelt, Autonomie und Selbstständigkeit wird durch Kreativität verwirklicht. Bei dem Spiel kann der Progression der Lernenden gefolgt werden, es kann auf immer höherem Sprachniveau eingesetzt werden. Es bietet die Möglichkeit, eine Palette unterschiedlicher Lexik und Situationen zu üben und zu beherrschen.

Teaching International Commercial Legal English to Students of Business Law

\section{Zielgruppe:}

Masterstudierende des Studiengangs Wirtschaftsrecht mit der Spezialisierung Internationales Recht, welche gleichzeitig einen Fachkurs in "International Commercial Transactions" belegen. Eine kleine Gruppe von bis zu 15 Studierenden, Niveaustufe Englisch B2-C1, jedoch ohne Kenntnisse der Fachsprache in diesem Lehrgebiet.

\section{Lernziele:}

Die Studierenden sind nach dem Kurs in der Lage, ein komplexes internationales Geschäft mit der dazugehörenden Dokumentation (Korrespondenz zwischen den beteiligten Unternehmen, einschlägige Dokumente - Kaufvertrag, AGB, Akkreditiv, Frachtbrief, usw.) in englischer Sprache nachzuvollziehen und in ihrer zukünftigen Berufspraxis mit ein wenig professioneller Hilfe selbst zu gestalten.

\section{Methode:}

Der Kurs wird in drei zeitliche Abschnitte geteilt. Einige Wochen lang beschäftigen wir uns im Seminarraum mit den Rahmenbedingungen und Dokumenten eines internationalen Import-Export-Geschäfts - aus inhaltlicher und sprachlicher Sicht. Es entstehen Synergien zu dem parallelen Fachkurs in „International Commercial Transactions“.

Nach einem guten Drittel des Semesters bilden die Studierenden Projektgruppen. Sie gründen fiktive Unternehmen, verhandeln ein Geschäft miteinander und führen es durch. Wo die Studierenden dies miteinander tun, ist ihnen freigestellt. Der Lehrende agiert als Coach, berät die Gruppen über inhaltliche und sprachliche Fragen - im Seminarraum, nach Terminvereinbarung, und per
E-Mail. Die Studierenden stellen eigene Forschungen an, um ein realistisches Geschäft miteinander abzuwickeln und geeignete Dokumente zu erstellen. Sie schicken ihre Dokumentation an den Trainer, der diese kommentiert und korrigiert und die Studierenden dabei durch Hinweise ermutigt, an der Korrektur ihrer „Fehler“ selbst mitzuwirken.

In der letzten Phase des Semesters präsentieren die Projektgruppen ihre Ergebnisse in Form einer Präsentation und eines Portfolios mit den Dokumenten und einer Selbsteinschätzung des eigenen Lernerfolgs.

\section{Kritisches Resumée:}

Diese semesterumfassendeProjektarbeit ist aktivierend und spielerisch zugleich: Studierende spielen (simulieren) ein wirkliches Geschäft mit komplexen Abläufen und sie tun dies, indem sie (Geschäfts-)Beziehungen miteinander eingehen. Viele Studierende blühen dabei auf, übernehmen Verantwortung für den Prozess und damit für eigenes Lernen. Dass der Lehrende als Coach fungiert, ist mit Sicherheit ein guter Ansatz, doch hier müssen Studierende ermutigt werden, diese Dienstleistung in vollem Maße in Anspruch zu nehmen. Ein Projekt dieser Komplexität eignet sich für höhere Semester und insbesondere für kulturell gemischte Gruppen, da dann ein zusätzlicher Realismus entsteht: Menschen aus verschiedenen Nationen verwenden die englische Sprache, um miteinander zu arbeiten. Lernen darf nicht ausschließlich im Klassenzimmer erfolgen.

\section{Die Lektüre von Theaterstücken als aktivierende Methode im DaF-Unterricht}

\section{Zielgruppe:}

Studierende aller Bachelor- und Masterstudiengänge, die den DaF-Oberstufenkurs (B2-C1) besuchen, aber eventuell auch schon Teilnehmende des DaF-Mittelstufenkurses (A2-B1). Die Gruppe sollte etwa 6-16 Studierende umfassen.

\section{Lernziele:}

Einerseits soll ein Anreiz geboten werden, sich über den Fremdsprachenkurs hinaus weiter eigenständig mit deutscher Literatur und Kultur zu beschäftigen. Andererseits bietet die sprachliche Form dramatischer Texte, der Dialog, und seine Umsetzung im darstellenden Spiel die Chance, die Scheu vor der Fremdsprache zu verlieren. Die Hemmschwelle, im Alltagsbereich in der Fremdsprache zu kommunizieren, kann auf diese Weise gesenkt werden. Schließlich werden die Studierenden bereits auf Rollenspiele wie etwa in den betriebswirtschaftlichen Fachsprachenkursen vorbereitet, in denen die Studierenden in Gruppen z. B. die Gründung einer Firma simulieren sollen.

\section{Methode:}

Der Textkorpus eines Dramas ist reduziert, die sprachliche Form, der Dialog, und die Möglichkeit der Bewältigung auf der Bühne haben zur Folge, dass die Texte syntaktisch einfacher gestaltet sind als in den erzählenden Gattungen und semantisch weniger opak sind als in der Lyrik. Dies sind gute Gründe dafür, ein Theaterstück als Einstieg in die Lektüre fremdsprachlicher Literatur zu wählen.

Tatsache ist aber auch, dass ein dramatischer Text ein aktiver, aktivierender Text ist, der eine performative Realisierung verlangt. Bei geeigneten Szenen des Stückes (geeignet, weil die Anzahl der auftretenden Personen beschränkt ist, es sich um Schlüsselszenen innerhalb des Dramas handelt, weil die Dialoge dem Sprechniveau der Studierenden am besten entsprechen etc.) kann der Kurs, nachdem das reine Textverständnis geklärt wurde und ohne dass die Studierenden über den weiteren Verlauf des Theaterstückes Bescheid wissen, in Gruppen geteilt werden, innerhalb der Gruppe entscheidet das Los über den ,Regisseur‘. Der ,Regisseur‘ verteilt unter den Gruppenmitgliedern die Rollen und beginnt dann mit den Proben zu seiner Inszenierung der ausgewählten Theaterszene. Die Gruppen kommen schließlich wieder zusammen und präsentieren einander ihre Inszenierungen. Die Studierenden werden feststellen, dass Schwerpunkte, vielleicht aber auch nur Nuancen unterschiedlich gesetzt werden, sich ihre Interpretationen der Szene ähneln oder deutlich unterscheiden. Abschließend wird zusammen die folgende Szene gelesen und darüber debattiert, welche der Inszenierungen nach dem aktualisierten Kenntnisstand über den weiteren Handlungsverlauf am treffendsten erscheint. 
Eine weitere Möglichkeit, in Gruppen an einem dramatischen Text zu arbeiten, ist es, den Studierenden eine neue Szene auszuhändigen, nicht jedoch den Beginn der Szene. Innerhalb der Gruppe müssen sich nun die Studierenden zunächst mit dem Inhalt der neuen Szene auseinandersetzen, und sollen dann, unter Rückgriff auf den Kenntnisstand des bisherigen Handlungsverlaufs, den fehlenden Anfang selbst verfassen. Die Gruppen stellen anschließend ihre Szenenanfänge vor und begründen ihre Vorschläge, bevor alle Studierenden den Originalszenenbeginn erhalten und mit ihren eigenen Textideen vergleichen können. Dieses Prozedere ähnelt Übungen im Bereich der technischen Studiengänge und der Logistik, in denen Studierende im Team Ursachenforschung betreiben und Lösungsstrategien für gegebene Problemstellungen entwickeln sollen.

\section{Kritisches Resumée:}

Literarische Texte sind selbstverständlich komplexer als jeder Lehrbuchtext. Während der Lehrende in den Oberstufenkursen eher die Rolle des Coach annehmen kann, der die Lektüre und das darstellende Spiel begleitet und nur in Ausnahmefällen interveniert, etwa um eine weiterführende Diskussion anzuregen, muss er in der Mittelstufe massiver erklärend eingreifen, schon um das bloße Textverständnis sicher zu stellen.

\section{LITERATUR}

Asendorpf, J. (1988): Keiner wie der andere - Wie Persönlichkeitsunterschiede entstehen, München.

Bruns, A. (2003): Partizipatorische Ansätze im Unterricht ür Deutsch als Fremdsprache,

http://www.freire.de/node/70 (abgerufen am 8.9.2012).

O'Donoghue, J. (2012): Task-Based Language Teaching A New Methodological Approach to Second Language Acquisition Opportunities and Challenges. In: Wissenschaftliche Beiträge, Heft 2012, TH Wildau [FH], 153-159.

Lehner, M., Ziep, K.-D. (1997): Phantastische Lernwelt, Vom „Wissensvermittler“ zum „Lernhelfer“, Weinheim.

Nolte, D. (2012): Auf den Lehrer kommt es an, Tagesspiegel 16.2.2012, 29

Roche, J. (2008): Fremdsprachenerwerb und Fremdsprachendidaktik, Tübingen.

Wahl, D. (2006): Lernumgebungen erfolgreich gestalten, vom trägen Wissen zum kompetenten Handeln, Bad Heilbrunn.
AUTOREN

Daniela Schultz, M. A.

Fachbereich Wirtschaft, Verwaltung und Recht

TH Wildau $[\mathrm{FH}]$

Telefon: +49 (0) 3375 / 508-262

daniela.schultz@th-wildau.de

Martin Bradbeer, MBA

Fachbereich Wirtschaft, Verwaltung und Recht TH Wildau [FH]

Telefon: +49 (0) 3375 / 508-401

bradbeer@th-wildau.de

Greg Bond, Dr.

Fachbereich Wirtschaft, Verwaltung und Recht TH Wildau [FH]

Telefon: +49 (0) 3375 / 508-366

bond@th-wildau.de

Virginie Bimont, M. A.

Fachbereich Wirtschaft, Verwaltung und Recht TH Wildau [FH]

Telefon: +49 (0) 3375 / 508-427 virginie.bimont@th-wildau.de

Adrian Stiglbauer, M. A.

Fachbereich Wirtschaft, Verwaltung und Recht TH Wildau [FH]

Telefon: +49 (0) 3375 / 508-20015

stiglbauer@th-wildau.de 